Original research article

\title{
A-769662 stimulates the differentiation of bone marrow- derived mesenchymal stem cells into osteoblasts via AMP-activated protein kinase-dependent mechanism
}

\author{
Basem M. Abdallah *, Abdullah M. Alzahrani \\ King Faisal University, College of Science, Biological Sciences Department, Al-Ahsa, Saudi Arabia
}

\begin{abstract}
AMP-activated protein kinase (AMPK) signaling shows an important role in energy metabolism and has recently been involved in osteogenic and adipogenic differentiation. In this study we aimed to investigate the role of AMPK activator, A-769662, in regulating the differentiation of mesenchymal stem cells derived from bone marrow (BMSCs) into osteoblastic and adipocytic cell lineage. The effect of A-769662 on osteogenesis was assessed by quantitative alkaline phosphatase (ALP) activity, matrix mineralization stained with Alizarin red, and gene expression analysis by quantitative polymerase chain reaction (qPCR). Adipogenesis was determined by Oil Red $\mathrm{O}$ staining for fat droplets and qPCR analysis of adipogenic markers. A-769662 activated the phosphorylation of AMPK $\alpha 1$ during the osteogenesis of mBMSCs as revealed by western blot analysis. A-769662 promoted the early stage of the commitment of mouse (m) BMSCs differentiation into osteoblasts, while inhibiting their differentiation into adipocytes in a dose-dependent manner. The effects of A-769662 on stimulating osteogenesis and inhibiting adipogenesis of mBMSCs were significantly eliminated in the presence of either $A M P K \alpha 1$ siRNA or Compound C, an inhibitor of AMPK pathway. In conclusion, we identified A-769662 as a new compound that promotes the commitment of BMSCs into osteoblasts versus adipocytes via AMPK-dependent mechanism. Thus our data show A-769662 as a potential osteo-anabolic drug for treatment of osteoporosis.
\end{abstract}

Keywords: A-769662; Adipocyte; AMPK; BMSC; Osteoblast; Stem cells

\section{Highlights:}

- The study shows the stimulatory effect of AMPK activator, A-769662 on the differentiation of mesenchymal stem cells into osteoblast.

- Data demonstrated the inhibitory effect of A-769662 on adipogenesis.

- A-769662 induces osteogenesis via AMPK dependent mechanism.

\section{Introduction}

Mesenchymal stem cells derived from bone marrow (BMSCs; known as skeletal stem cells) are a group of adult stem cells that share the same microenvironment with hematopoietic stem cells in the bone marrow niche. BMSCs are known to have both self-renewal capacity and differentiate potential which falls into three-mesoderm lineages including adipocytes, osteoblasts and chondrocytes (Abdallah and Kassem, 2008; Abdallah et al., 2015; Bianco and Robey, 2015). Preclinical and clinical studies showed the potential role of BMSCs in promoting bone and dental regeneration after trauma in bone diseases, including osteoporosis, dental, facial and long bone defects (Luby et al., 2019; Zheng et al., 2019).

Several intracellular kinase pathways regulate the stage specific differentiation of BMSCs into osteoblasts. These in- clude: transforming growth factor $\beta$ (TGF $\beta$ ) activated kinase 1 (TAK1) (Chen et al., 2012); phosphoinositide 3-kinase (PI3-kinase)/Akt (Amantea et al., 2008); AMP-activated protein kinase (AMPK) (Kanazawa et al., 2009; Kim et al., 2012; Shah et al., 2010); and mitogen-activated protein kinase (MAPK)/ extracellular signal-regulated kinase (ERK) (Abdallah et al., 2015; Ge et al., 2007; Kanno et al., 2007). Thus, identifying compounds that could target any of these osteogenic-specific protein kinases will promote the differentiation of BMSC s into osteoblasts.

AMPK is a serine/threonine protein kinase complex, it consists of $\alpha, \beta$ and the regulatory $\gamma$ subunits. AMPK has been reported to regulate cellular energy metabolism (Hardie, 2014; Mihaylova and Shaw, 2011; Wang et al., 2012), cellular autophagy (a caspase-independent cell degradation process), and cell cycle arrest (Tamargo-Gomez and Marino, 2018). In this context, several reports have investigated AMPK as a novel

\footnotetext{
* Corresponding author: Basem M. Abdallah, Associate Professor, King Faisal University, College of Science, Biological Sciences Department, P.O Box 380, Al-Ahsa, 31982, Saudi Arabia; e-mail: babdallallah@kfu.edu.sa http://doi.org/10.32725/jab.2021.016

Submitted: 2020-12-30 • Accepted: 2021-06-21 • Prepublished online: 2021-07-01

J Appl Biomed 19/3: 159-169 • EISSN 1214-0287 • ISSN 1214-021X

(c) 2021 The Authors. Published by University of South Bohemia in České Budějovice, Faculty of Health and Social Sciences.

This is an open access article under the CC BY-NC-ND license.
} 
therapeutic target for type 2 diabetes, atherosclerosis, obesity and cancer (Babkov et al., 2019; Day et al., 2017; Thirupathi and Chang, 2019).

Thienopyridone (A-769662) is an established AMPK activator that allosterically activates AMPK without affecting kinases upstream of the AMPK $\beta$ subunit (Kim et al., 2016). A-769662 was found to suppress adipogenesis in a pre-adipocyte cell line (Zhou et al., 2009), reduce body weight gain and decrease plasma glucose in ob/ob mice (Cool et al., 2006), and inhibit mesenchymal stem cell (MSC) proliferation in a p27-dependent manner (de Meester et al., 2014). Additionally, A-769662 reportedly protects osteoblasts against hydrogen peroxide $\left(\mathrm{H}_{2} \mathrm{O}_{2}\right)$-induced apoptosis ( $\mathrm{Zhu}$ et al., 2014). However, the effect of A-769662 on BMSC differentiation into osteoblasts has not yet been reported.

In this study, we investigated the effect of A-769662 on BMSCs differentiation into osteoblasts versus adipocytes. Interestingly, A-769662 significantly stimulated osteogenesis while suppressing adipogenesis of BMSCs via an AMPK-dependent mechanism.

\section{Materials and methods}

\section{Cell cultures and reagents}

Primary mBMSCs were isolated from male C57BL/6J mice as previously described (Abdallah et al., 2018). Animals were treated according to the national and institutional guidelines for the ethical and legal treatment of animals. The Standing Research Ethics Committee, King Faisal University, Saudi Arabia, approved the extraction of BMSCs from mice. For BMSCs isolation, mice were sacrificed at the age of 2 months, and this was followed by immediate dissection. In brief, cells flushed out from the bone marrow of mouse tibia and femur were filtrated using $70 \mu \mathrm{m}$ nylon mesh filter and cultured in RPMI1640 medium supplemented with $12 \mu \mathrm{M}$ L-glutamine (Invitrogen), $1 \%$ penicillin/streptomycin (P/S) (Gibco Invitrogen, USA), and $12 \%$ FBS (Gibco Invitrogen). Non-adherent cells were removed after $24 \mathrm{~h}$, washed and cultured in the above medium at $37^{\circ} \mathrm{C}, \mathrm{CO}_{2}$ incubator.

Human BMSC cells were purchased from Cell Applications Inc. (San Diego, CA). hBMSCs were cultured in DMEM medium (Dulbecco's modified Eagle medium) (Sigma-Aldrich) containing $1 \%$ penicillin/streptomycin and 10\% FBS (Gibco Invitrogen), according to the manufacturer's instructions. Medium was changed every 2-3 days.

Compound $C$ and A-769662 were purchased from Calbiochem (Darmstadt, Germany) and Cayman Chemical (Ann Arbor, MI, USA) respectively.

\section{Cell toxicity assay}

MTT cell proliferation assay kit (Sigma-Aldrich) and CellTiter-Blue ${ }^{\circledR}$ Cell Viability Assay (Promega, USA) were used to evaluate the cell viability according to the manufacturer's instructions kit. Cells were incubated with MTT solution to metabolize to formazan and with CellTiter-Blue ${ }^{\circledR}$ solution to reduce resazurin to resorufin. Absorbance was measured at a wavelength of $550 \mathrm{~nm}$ for MTT assay and at $579 \mathrm{~nm}$ for CellTiter-Blue ${ }^{\circledR}$ assay.

\section{Differentiation of BMSCs into osteoblasts}

mBMSCs were stimulated to differentiate into osteoblasts using osteogenic induction medium (OIM) containing a-minimum essential medium ( $\alpha$-MEM; Gibco) supplemented with $100 \mathrm{U} / \mathrm{ml}$ of penicillin/streptomycin (P/S), 10\% FBS, $10 \mathrm{mM}$ $\beta$-glycerol-phosphate (Sigma-Aldrich ApS), and $50 \mathrm{mg} / \mathrm{ml}$ of vitamin C (Sigma-Aldrich ApS).

\section{Differentiation of BMSCs into adipocytes}

mBMSCs were stimulated to differentiate into adipocytes with DMEM supplemented with $100 \mathrm{U} / \mathrm{ml}$ of $\mathrm{P} / \mathrm{S}, 9 \%$ horse serum, $250 \mathrm{nM}$ dexamethasone, $450 \mu \mathrm{M}$ 1-methyl-3-isobutylxanthine (IBMX), $1 \mu \mathrm{M}$ rosiglitazone (BRL 49653, Cayman Chemical, USA), and $5 \mu \mathrm{g} / \mathrm{ml}$ insulin (Sigma-Aldrich).

\section{Measurement of alkaline phosphatase (ALP) activity} mBMSCs were cultured and induced with OIM in 96 well plate. The quantification of ALP activity was measured by incubating the cells with P-nitrophenyl phosphate $(1 \mathrm{mg} / \mathrm{ml})$ at $37{ }^{\circ} \mathrm{C}$ for $20 \mathrm{~min}$. The reaction was terminated by $3 \mathrm{M} \mathrm{NaOH}$. The absorbance was measured at $405 \mathrm{~nm}$. CellTiter-Blue ${ }^{\circledR}$ cell viability assay was used to determine cell viability. Samples were measured in 6 replicates. Values were normalized to cell viability and represented as fold change over control including non-induced cells.

\section{Cytochemical staining}

ALP staining

mBMSCs were stimulated to differentiate into osteoblasts as mentioned above. Cells were fixed in acetone/citrate buffer $\mathrm{pH} 4.2(1.5: 1)$ at RT for $5 \mathrm{~min}$. Staining was performed in Napthol-AS-TR-phosphate solution (Sigma-Aldrich ApS) at RT for 1 h. Napthol-AS-TR-phosphate is diluted 1 : 5 in $\mathrm{H}_{2} \mathrm{O}$ and mixed $1: 1$ with Fast Red TR (Sigma-Aldrich ApS) diluted $1: 1.2$ in 0.1 M Tris buffer, $\mathrm{pH} 9.0$.

\section{Quantification of Alizarin Red S staining}

mBMSCs were stimulated with OIM for 10-12 days. Cells were fixed with $70 \%$ ice-cold ethanol at $-20{ }^{\circ} \mathrm{C}$ for one hour. The mineralized matrix produced by the cells upon osteoblast differentiation was stained with Alizarin red solution (Sigma-Aldrich ApS, $40 \mathrm{mM}, \mathrm{pH}=4$ ) at RT for $10 \mathrm{~min}$. For quantification of mineralization, cells stained with AR-S were incubated with $10 \%$ cetylpyridinium chloride (Sigma-Aldrich $\mathrm{ApS}$ ) at RT for 1 hour. The eluted dye was measured spectrophotometrically at $570 \mathrm{~nm}$.

Quantification of lipid accumulation by Oil Red O staining mBMSCs were induced to differentiate into adipocytes as mentioned above. After one week of induction, cells were fixed in $4 \%$ paraformaldehyde at RT for $10 \mathrm{~min}$, and lipid droplets were stained with Oil Red O solution (prepared by dissolving $0.5 \mathrm{~g}$ in $60 \%$ isopropanol) (Sigma-Aldrich). For lipids quantification, Oil Red O was eluted with isopropanol for $10 \mathrm{~min}$ at RT. The extracted dye was measured spectrophotometrically at OD $490 \mathrm{~nm}$. Values were normalized to the number of viable cells and represented as fold change over control.

\section{RNA extraction and real-time PCR analysis}

Total RNA was isolated from cells using TRIzol (Thermo Fisher Scientific, Roskilde, Germany). $1 \mu \mathrm{g}$ of total RNA was used to synthesize cDNA using the RevertAid $\mathrm{H}$ minus first strand cDNA synthesis kit (Fermentas, St Leon-Rot, Germany). The qPCR was performed using Fast SYBR $^{\circledR}$ Green Master Mix in Applied Biosystems 7500 Real-Time system (Applied Biosystems, California, USA). Suppl. Table 1 shows the primer sequences used. The expression value of each target gene was normalized to the mRNA expression values of reference genes $\beta$-Actin and Hprt, using a comparative CT method [(1/(2delta-CT) formula] with Microsoft Excel $2007^{\circledR}$ (Abdallah et al., 2018). 


\section{Western blot analysis}

Cells were lysed in cell lysis buffer (10 mM Tris-HCl, pH 7.4, $150 \mathrm{mM}$ sodium chloride, 1\% NP-40, 0.1\% SDS, 1 mM EDTA, $1 \mathrm{mM}$ phenyl-methylsulfonyl fluoride, $1 \mathrm{mM} \mathrm{NaF}, 1 \mathrm{mM}$ $\mathrm{Na}_{3} \mathrm{VO}_{4}$ ), supplemented with protease inhibitor cocktail (Roche Diagnostics, Mannheim, Germany). $20 \mu \mathrm{g}$ of protein was separated on $8-12 \%$ NuPAGE$^{\circledR}$ Novex $^{\circledR}$ Bis-Tris gel systems (Thermo Fisher Scientific, Germany) followed by transfer to PVDF membrane (Millipore, USA). The membrane was blocked and probed with antibodies (dilution $1: 1000$ ) and incubated with secondary anti-rabbit horseradish peroxidase-conjugated antibody (Santa Cruz Biotechnology, Heidelberg, Germany). Antibodies against total and phospho AMPK $\alpha 1$ (Thr172), p-ACC (Ser79), ERK, p38, AKT and Smad 1/5/8 were purchased from Cell Signalling Tech. (Denver, MA, USA). Total Smad1/5/8 was purchased from Santa Cruz Biotechnology, Inc. (Heidelberg, Germany) and a-tubulin from Sigma (USA).

\section{siRNA transfection of $\mathrm{mBMSCs}$}

The sequence of siRNA used for silencing $A M P K \alpha 1$ was, GCAUAUGCUGCAGGUAGA UdTdT (Thermo Fisher Scientific). As a negative control, the non-targeting control siRNA was used. The reverse transfection protocol was used to transfect the mBMSCs with $100 \mathrm{nM}$ siRNA using Lipofectamine 2000 (ThermoFisher Scientific) as described previously (Jafari et al., 2015).

\section{Statistical analysis}

All values are presented as the mean value \pm SD (standard deviation) of 3 values of 3 independent experiments. Unpaired Student's T-test (2-tailed) was used, assuming equal variation in the two groups. Differences were considered statistically significant at ${ }^{*} P<0.05$, and ${ }^{* *} P<0.005$.

\section{Results}

\section{AMPK activation in primary $m B M S C s$ by $A-769662$}

We studied the cytotoxic effect of A-769662 on mice (m) BMSCs. We measured the cell viability of the primary culture of mBMSCs in the presence of different A-769662 doses using the MTT assay and CellTiter-Blue. A-769662 exerted a toxic effect on cell viability at concentrations above $10 \mu \mathrm{M}$ (Fig. 1A, 1B). Therefore, A-769662 was used at concentrations between $0.1-10 \mu \mathrm{M}$ throughout this study. Further, we examined AMPK signaling activation by A-769662 in mBMSCs over a 6-day culture period. Treatment of mBMSCs with A-769662 significantly activated AMPK signaling, as revealed by the activation of the phosphorylation of AMPKa and its substrate p-acetyl CoA carboxylase (ACC) via western blot assay (Fig. 1C). To examine, whether A-769662 is targeting any other known osteogenic signaling pathways, we studied the effect of A-769662 on the activation of ERK, p-38, AKT and Smad 1/5/8 proteins. Interestingly, treatment of mBMSCs with A-769662 did not show any significant effect on the phosphorylation of the above proteins during osteogenesis as compared to non-treated cells (Suppl. Fig. 1A). In addition, A-769662 did not promote the stimulatory effect of any known signaling molecules (including TGF $\beta 1$, BMP2, Wnt3a, bFGF, and PDGF) on osteogenesis in mBMSCs (Suppl. Fig. 1B). This finding confirmed the specificity of A-769662 in targeting AMPK signaling during osteogenesis.

\section{(A)}

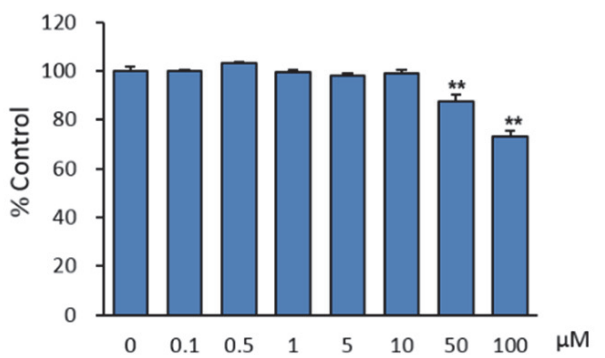

(B)

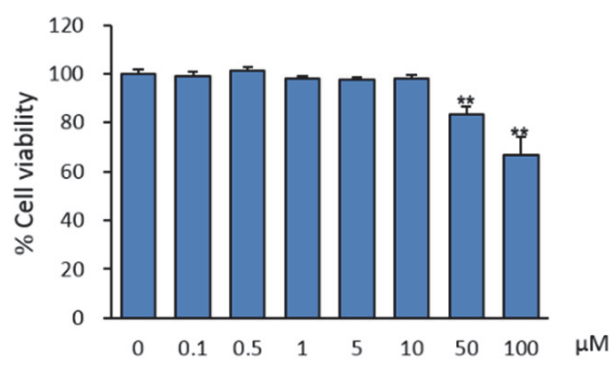

(C)

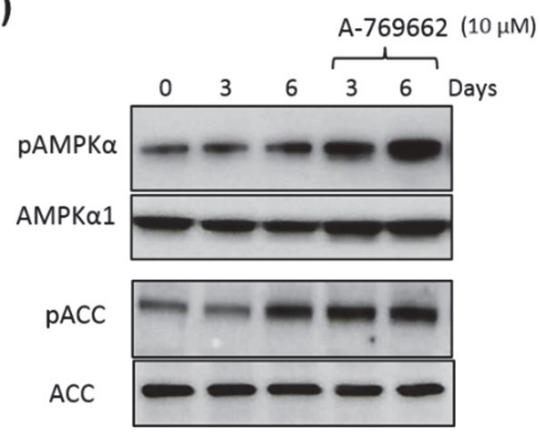

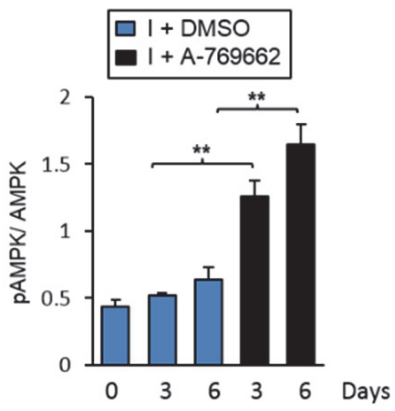

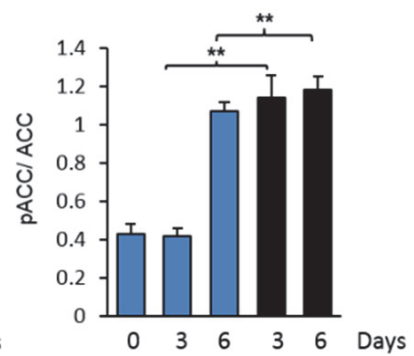

$(10 \mu \mathrm{M})$

Fig. 1. Activation of AMPK in mBMSCs by A-769662. (A) Cytotoxicity of A-769662 on mBMSCs as measured by MTT assay and CellTiter-Blue ${ }^{\circledR}$ assay (B). Cells were treated either with DMSO (0) as control or different concentrations of A-769662 for 3 days in culture. (C) Western blot analysis for activation of AMPK by A-769662 $(10 \mu \mathrm{M})$ in mBMSCs. Values for each phospho protein normalized to corresponding total protein and are presented in the graphs. Values are mean \pm SD of triplicates; ${ }^{* *} p<0.005$ compared to control cells with DMSO. 


\section{A-769662 promotes the differentiation of $m B M S C s$ into osteoblasts}

The effect of A-769662 was examined on osteoblast differentiation of mBMSCs. ALP activity quantification revealed the stimulatory effect of A-769662 on the osteoblast differentiation of mBMSCs in a dose-dependent manner (Fig. 2A). In addition, quantification of matrix mineralization by Alizarin Red staining after 12 days of induction was significantly and dose dependently increased by A-769662 treatment (Fig. 2B). At the molecular level, treatment of mBMSCs with A-769662 for 12 days during osteogenesis, significantly upregulated the mRNA expression of the osteoblast master transcription regulator, Runx2, and osteoblast-related genes, including Alp, Col1a1, Ocn and Opn by ( $\geq 1.5$-fold; Fig. 2C).

\section{A-769662 promotes early commitment of mBMSCs into osteoblasts}

To understand the mechanism of A-769662-induced osteogenesis, we treated mBMSCs with A-769662 at different osteoblast differentiation stages: early commitment (days 0-3), extracellular matrix development (days 3-9) and matrix mineralization (days 9-12). ALP activity and Alizarin red quanti- fication were performed on day 12. As shown in Fig. 2D, treatment of mBMSCs with A-769662 at both early (days 0-12) and middle stages (days 3-12) increased ALP activity by $98 \%$ and $63 \%$ respectively, while treatment during the late middle stage and late stage (days 6-12 and days 9-12) did not alter ALP activity. This effect was further supported by quantifying matrix mineralization during the same treatment strategies after 12 days of induction (Fig. 2D, E). We also evaluated the effect of AMPK inhibition on osteogenesis using Compound $C$ (Comp. C), a potent AMPK inhibitor. Treatment of mBMSCs with $10 \mu \mathrm{M}$ Comp. C significantly blocked AMPKa phosphorylation and its substrate (ACC), as shown in Fig. 3A. Comp. C supressed the mBMSCs differentiation into osteoblasts, as denoted by a significant decrease in ALP activity and Alizarin red quantification by $62 \%$ and $57 \%$, respectively, compared to control cells treated with DMSO (Fig. 3B, C). Furthermore, siRNA-mediated silencing of $A M P K \alpha 1$ (siAMPK $\alpha 1$ ) suppressed the $A M P K \alpha 1$ mRNA expression in mBMSCs by $82 \%$ (Fig. 3D). Silencing of $A M P K \alpha 1$ showed to inhibit the mBMSCs differentiation into osteoblasts as measured by reduced ALP activity by $38 \%$ compared to siRNA control cells (Fig. 3E).
(A)

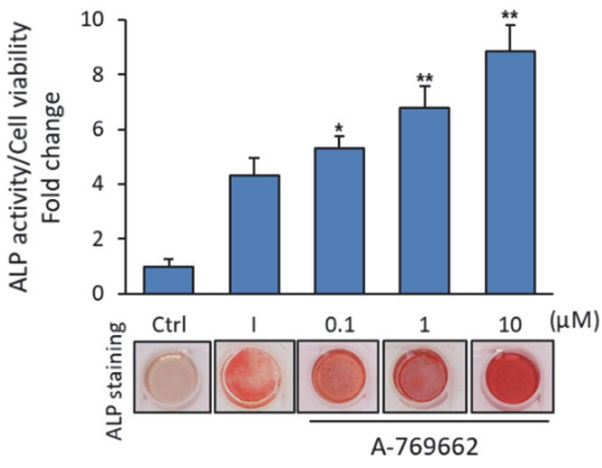

(B)

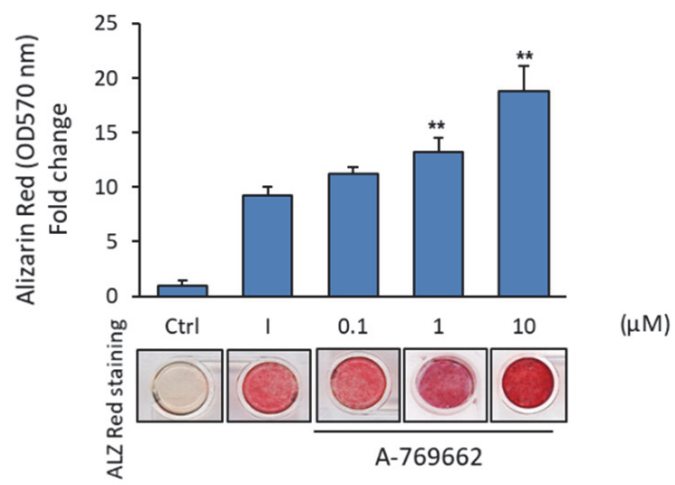

(C)

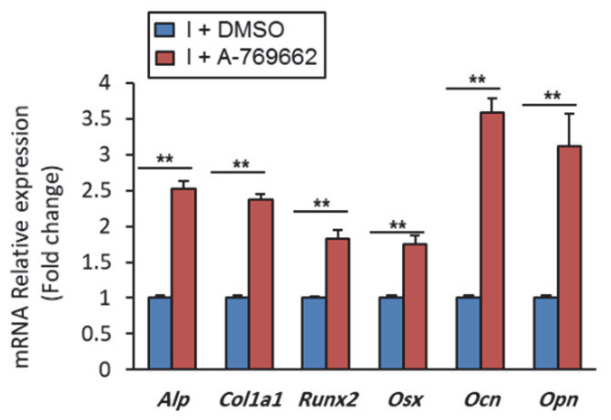

(D)

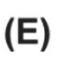

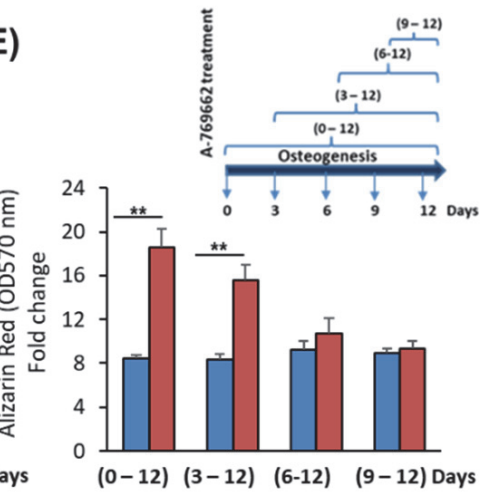

Fig. 2. A-769662 stimulates mBMSCs differentiation into osteoblasts. (A) Stimulatory effect of A-769662 on mBMSCs differentiation into osteoblast as assessed by quantification of ALP activity and (B) matrix mineralization stained with Alizarin red after osteogenic induction for 7 and 12 days respectively. mBMSCs were induced with osteogenic medium without (I+DMSO) or with different concentrations of A-769662. Images for staining were shown. (C) Osteogenic mRNA expression analysis by qPCR in mBMSCs treated without or with A-769662 (10 $\mu$ M) during osteoblast induction for 12 days. Expression values were normalization to reference genes and presented as fold change. (D) Effect of A-769662 on mBMSCs at different stages during osteogenesis, measured by ALP activity and (E) Alizarin Red staining. Cells were induced to differentiate into osteoblasts and then treated without or with A-769662 (10 $\mu \mathrm{M})$ at different time points (day 0, 3, 6, and 9) as shown by the schematic timeline. Values are mean \pm SD of triplicates; ${ }^{*} p<0.05,{ }^{* *} p<0.005$ compared to induced cells without A-769662. 
(A)

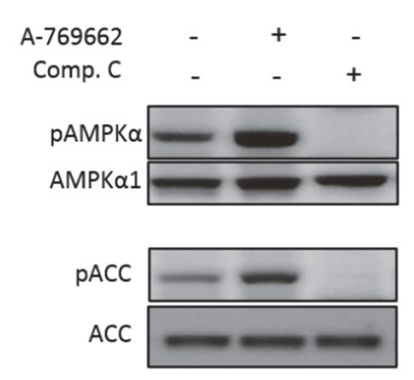

(D)

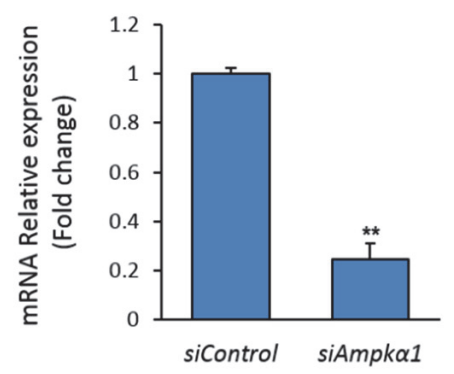

(B)
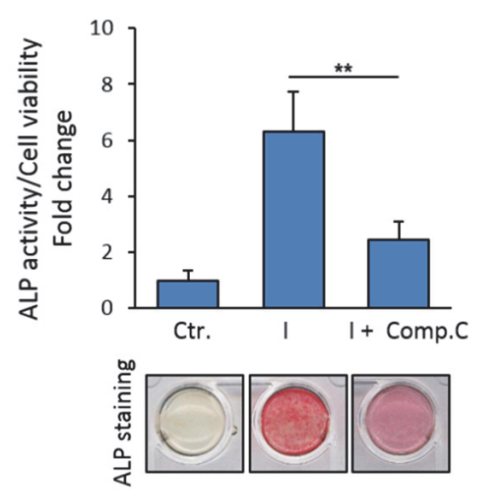

(E)

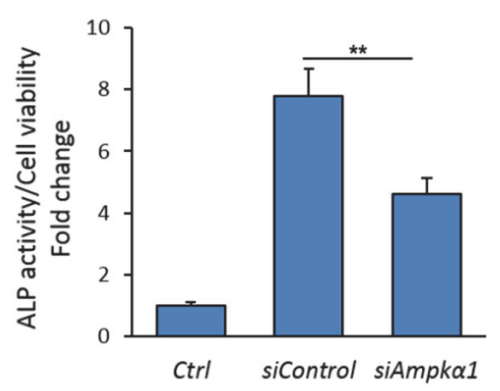

(C)

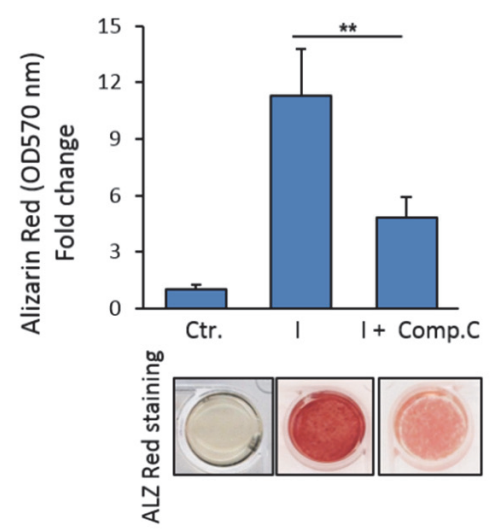

Fig. 3. A-769662 promotes early commitment of mBMSCs in osteoblast lineage. (A) Analysis of the inactivation of AMPKa and ACC phosphorylation in mBMSCs with Compound C (Comp. C) $(10 \mu \mathrm{M})$ by Western blot. (B) Comp. C suppressed the osteoblast differentiation of mBMSCs as shown by reduced ALP activity and (C) Alizarin red staining. Cells were analyzed after 7 days and 12 days of induction respectively. (D) mRNA expression analysis of Ampk 1 in mBMSCs transfected without (siControl) or with Ampk 1 siRNA (siAmpk 1 ). The qPCR was performed 6 days post transfection. (E) Effect of Ampk $\alpha 1$ siRNA on mBMSCs differentiation measured by quantitative ALP activity after 7 days of induction. Cells were reverse transfected with siRNA, and after $24 \mathrm{~h}$, the cells were induced to differentiate; ${ }^{* *} p<0.005$ compared to $\mathrm{I}+\mathrm{DMSO}$. Values are mean \pm SD of triplicates.

\section{A-769662 inhibits the mBMSCs differentiation into adipocytes}

A-769662 dose dependently inhibited the differentiation of mBMSCs into adipocyte, as quantified by Oil red $\mathrm{O}$ staining for lipid accumulation (Fig. 4A). qPCR analysis consistently demonstrated the inhibitory effect of A-769662 on mRNA expression of adipocyte-specific markers for both early (Ppary2 and $\mathrm{C} / e b p \alpha$ ) and late ( $a \mathrm{P} 2$ and $L p l$ ) genes in mBMSCs (Fig. 4B). On the other hand, AMPK inhibition by Comp. $C$ significantly stimulated adipogenesis, as assessed by increased lipid accumulation and expression of adipogenic mRNA markers after 12 days of induction (Fig. 4C, D). Similarly, transfection of mBMSCs with siAMPK $\alpha 1$ showed to stimulate their adipogenesis significantly as revealed by increased Oil red $O$ staining for fat droplets by $42 \%$ compared to siRNA control cells (Fig. 4E).

\section{AMPK activation was essential for A-769662-induced osteogenesis in mBMSCs}

To investigate the involvement of AMPK in mediating A-769662-induced osteogenesis, we measured the effect of A-769662 on osteogenesis and adipogenesis of mBMSCs pre-treated with Comp. C. A-769662-induced osteogenesis was eliminated by Comp. $C$ in $\mathrm{mBMSC}$, as shown by decreased levels of ALP activity after 7 days of osteogenic induction (Fig. 5A).
Similarly, A-769662-inhibited adipogenesis was rescued by Comp. $C$ treatment, as denoted by Oil red $\mathrm{O}$ staining after 12 days of induction (Fig. 5B). Thus, AMPK activation is required to mediate the regulatory effect of $A-769662$ on the differentiation of mBMSCs.

The activation of AMPK was reported to regulate the differentiation of BMSCs into osteoblast versus adipocyte by upregulating Runx2 and suppressing Ppary2 expression, the two main transcription factors for osteogenesis and adipogenesis respectively (Kim et al., 2012). Thus, we studied the regulation of Runx2 and Ppary2 expression as downstream targets of A-769662-induced osteogenesis. As shown in Fig. 5C, the stimulatory effect of A-769662 on Runx2 expression during osteogenesis was significantly reduced by Comp. C. On the other hand, the inhibitory effect of A-769662 on Ppary2 mRNA expression during adipogenesis of mBMSCs was rescued by Comp. C (Fig. 5D). Thus, A-769662 regulates the differentiation of mBMSCs into osteoblast or adipocyte by controlling the expression of downstream transcription factors, Runx2 and Ppary2.

\section{A-769662 stimulates the hBMSCs differentiation into osteoblast versus adipocyte}

Similar to its effect on mBMSCs, A-769662 induced an osteoblast lineage commitment for hBMSCs, as denoted by a sig- 
(A)

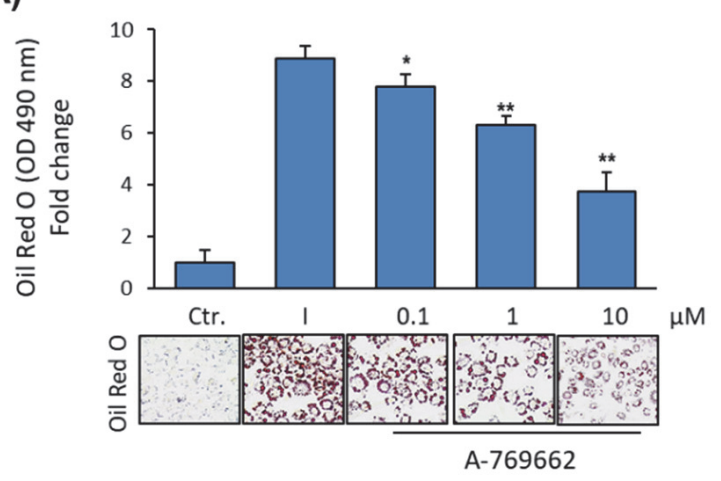

(C)

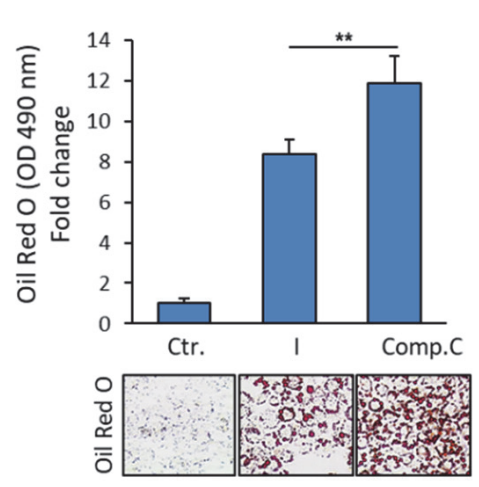

(B)
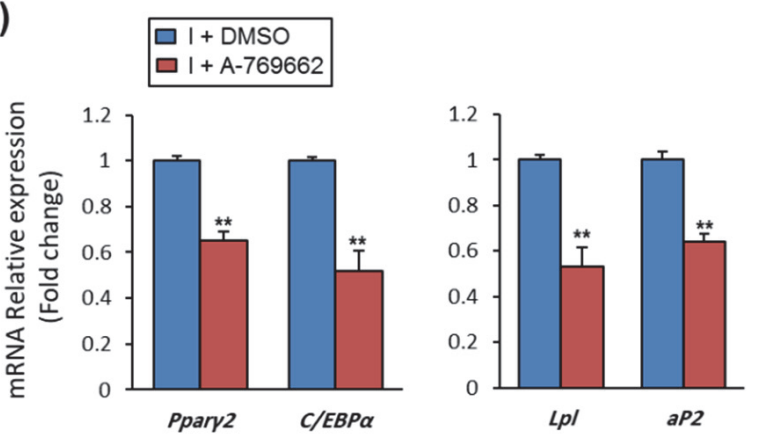

(D)

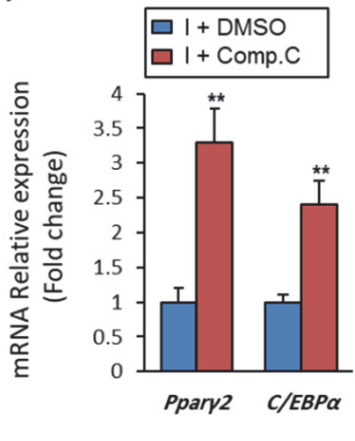

(E)

Fig. 4. A-769662 suppresses the differentiation of mBMSCs into adipocytes. (A) The inhibitory effect of A-769662 on adipocyte differentiation of mBMSCs as shown by quantitative Oil red O staining. Cells were induced to differentiate and then treated without (I+DMSO) as control or with different concentrations of A-769662 $(10 \mu \mathrm{M})$ for 12 days. Staining images with Oil red O were shown. (B) mRNA expression of adipogenic markers by qPCR analysis at day 12 of mBMSCs differentiation with or without A-769662 $(10 \mu \mathrm{M})$. (C) Stimulatory effect of Compound C on adipocyte differentiation as assessed by Oil red O staining and (D) upregulation of mRNA expression of adipogenic markers. (E) Stimulatory effect of Ampk $\alpha 1$ siRNA on adipogenesis of mBMSCs as quantified by Oil red O staining. Values are mean \pm SD of triplicates; ${ }^{*} p<0.05$, $*^{* *} p<0.005$ compared to control cells.

nificant dose-dependent increase in ALP activity after 7 days of osteogenic induction (Fig. 6A). In addition, A-769662 upregulated the expression of Runx2 in mBMSCs in a dose dependent manner (Fig. 6B). On the other hand, A-769662 dose dependently suppressed hBMSC differentiation into adipocytes in association with the downregulation of Ppary2 gene expression, as assessed by quantification of Oil Red O staining (Fig. 6C) and qPCR analysis (Fig. 6D) respectively.

\section{Discussion}

In this study, we demonstrated the dual role of an AMPK agonist, A-769662, in stimulating the early commitment of mBMSC differentiation into osteoblasts versus adipocytes. A-769662 exerted these effects via an AMPK-dependent mechanism and regulating the gene expression of Runx2 and Ppary2.

Several reports demonstrated the direct and specific effect of A-769662 on the activation of AMPK (Day et al., 2017; Kim et al., 2016). A-769662 was found to allosterically activate $\alpha \beta \gamma$ complex of AMPK and stabilizing the conformation of AMPK that resulted in the inhibition of dephosphorylation of Thr-172 in the AMPKa subunit (Sanders et al., 2007). In another mechanism, Ser108 phosphorylation in the AMPK $\beta 1$ subunit was shown to be required for the activation of AMPK by A-769662 (Scott et al., 2014). In addition, A-769662 was reported to activate AMPK without affecting the upstream kinases (Goransson et al., 2007).

Our data showed the stimulatory effect of A-769662 on the early commitment of mBMSCs differentiation into osteoblasts by upregulating the Runx2 transcription factor and its downstream target genes that encode matrix proteins. Several in vitro and in vivo studies reported the stimulatory effect of AMPK activation on osteogenic differentiation (Kanazawa et al., 2009; Molinuevo et al., 2010; Shah et al., 2010). Consistent with our findings, activation of AMPK showed to promote osteogenesis by regulating Runx2 mRNA expression (Kim et al., 2012) and phosphorylating RUNX2 (Chava et al., 2018). In addition, the AMPK activator, 5-aminoimidazole-4-carboxamide1-beta-d-ribonucleoside (AICAR) promoted osteoblast differentiation of osteoblastic MC3T3-E1 cells via inhibition of Rho-associated protein kinase (ROCK) (Kanazawa et al., 2009). AICAR and metformin, AMPK activators dose dependently increased the formation of trabecular bone nodule in rat calvaria-derived osteoblasts (Shah et al., 2010). Furthermore, in vivo metformin administration increased bone formation and suppressed the anti-osteogenic effect of rosiglitazone in rats (Molinuevo et al., 2010; Sedlinsky et al., 2011). In contrast, suppression of AMPK using either Comp. $C$ or short 
(A)

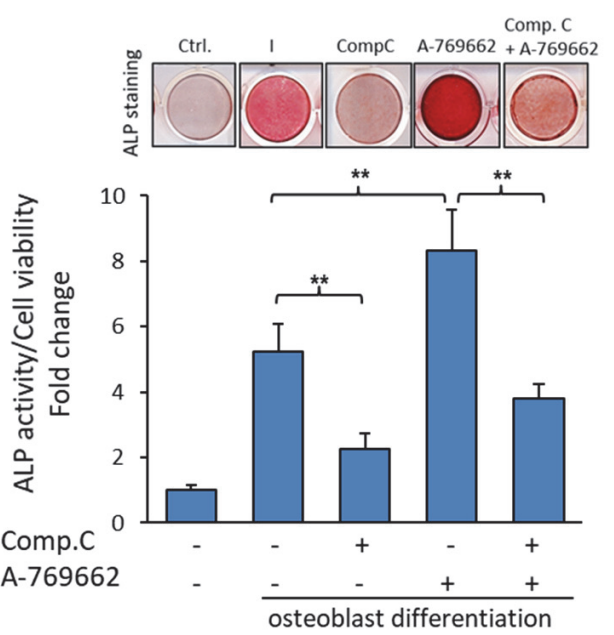

(C)

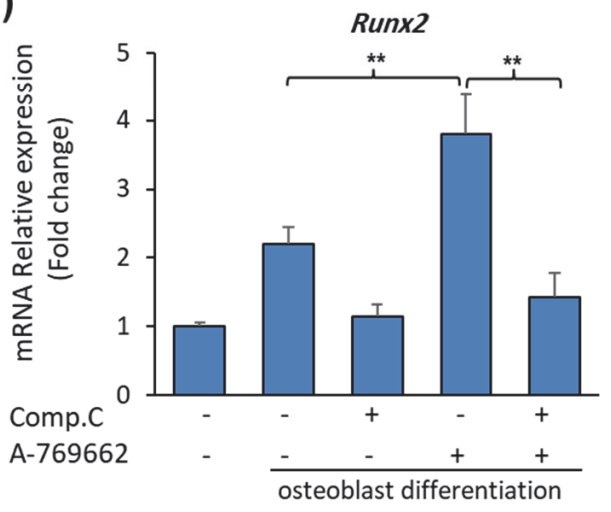

(B)

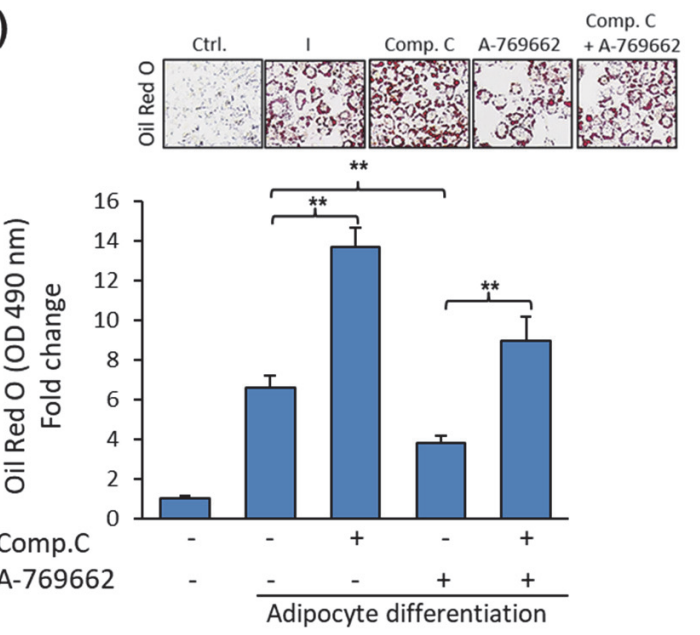

(D)

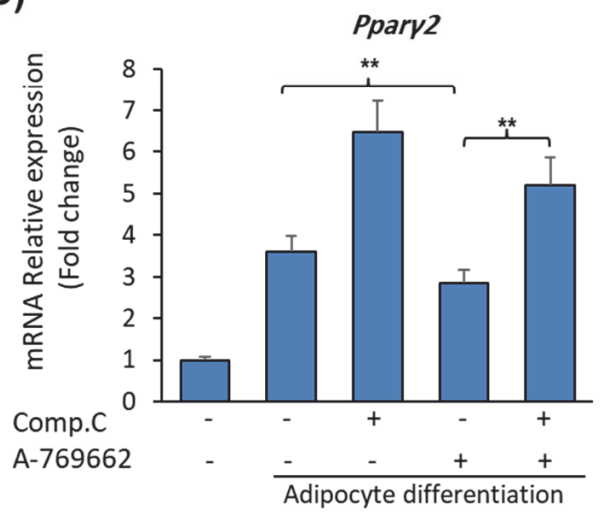

Fig. 5. The regulatory effect of A-769662 mBMSCs differentiation is mediated via AMPK-dependent mechanism. (A) The stimulatory effect of A-769662 on osteogenesis eliminated by Comp. C. ALP activity was determined in cells induced to osteoblastic lineage in the presence of Comp. $C(10 \mu \mathrm{M})$ without or with A-769662 for 7 days. ALP staining images are presented. (B) The inhibitory effect of A-769662 on adipogenesis is rescued by Comp. C. Oil red O staining quantification performed in induced mBMSCs to adipocytes in the presence of Comp. $C$ without or with A-769662 for 12 days. (C) qPCR analysis of Runx2 gene expression in mBMSCs under the same experimental condition of panel A. (D) qPCR analysis of Ppary 2 in mBMSCs under the same experimental condition of panel $\mathrm{B}$; $^{* *} p<0.005$ compared to I+DMSO. Each experimental value is mean \pm SD of independent triplicates.

hairpin RNA (shRNA)-mediated AMPK knockdown showed to inhibit osteogenesis by reducing their matrix mineralization and down-regulating the expression of Runx2 expression (Kim et al., 2012).

We demonstrated the inhibitory effect of A-769662 on the adipocyte differentiation of mBMSCs. A-769662 was consistently shown to inhibit adipocyte differentiation of 3T3-L1 (Zhou et al., 2009) and C3H10T1/2 cell lines (Chen et al., 2017). Additionally, A-769662 displayed anti-adipogenic effects in vivo by protecting mice against high fat diet-induced obesity (Wu et al., 2018) and reducing levels of both plasma and liver triglyceride in ob/ob mice (Cool et al., 2006).

Our finding that the effect of A-769662 on regulating BMSCs differentiation is mediated through an AMPK-dependent mechanism is consistent with the reported mode of action of A-769662 in controlling some physiological processes. For example, the inhibitory effect of A-769662 on adipogenesis in 3T3-L1 cells (Zhou et al., 2009) was associated with protection against $\mathrm{H}_{2} \mathrm{O}_{2}$-induced apoptosis (Zhu et al., 2014).

Several clinical studies have demonstrated the association of increased bone marrow fat mass with reduced bone mass in osteoporotic patients (Hardouin et al., 2016; Rharass and Lucas, 2018; Shen et al., 2012). This phenomenon is elucidated by the existence of an inverse relationship between osteoblast and adipocyte differentiation of BMSCs (Gimble et al., 2006; Li et al., 2018; Taipaleenmaki et al., 2011). In this context, several signalling pathways were found to be involved in enhancing the commitment of BMSCs into osteoblasts on the expenses of adipocytes. These include, canonical and non-canonical Wnt signalling, Sonic hedgehog and TGF- $\beta 1$ (Bennett et al., 2005; Zhao and Hantash, 2011).

A-769662 has a privilege as a direct and specific activator of AMPK, while other activators, like AICAR and metformin, that are used as drugs for cardiac ischemic injury (Corton et al., 1995) and diabetes (Towler and Hardie, 2007) were shown to function indirectly. For example, AICAR activator is a pro-drug that must be converted into the AMP analogue ZMP (Corton et al., 1995), and metformin showed to activate AMPK by increasing cellular AMP via inhibiting the mitochondrial respiratory chain complex I (Hardie, 2007). Thus, a drug that activates the AMPK directly and specifically like A-769662 would be more efficient in the treatment of metabolic diseases. However, more in vivo studies are needed to provide preclinical data on the therapeutic effect of A-769662 in disease animal models.

In this study, our data identified A-769662, an AMPK activator, as a novel compound that can plausibly be used as a the- 
(A)

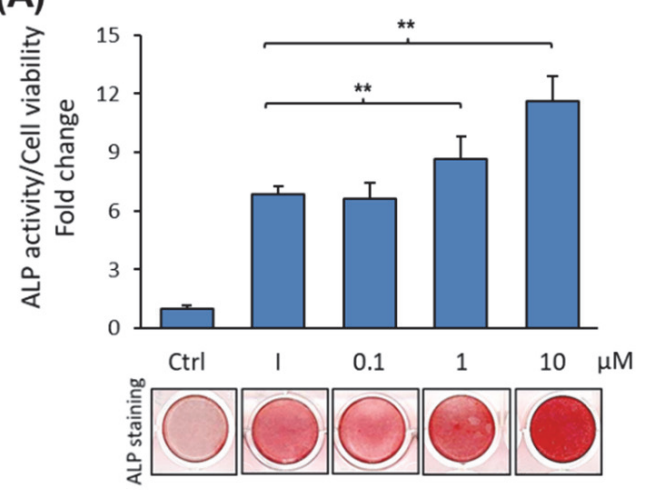

(C)

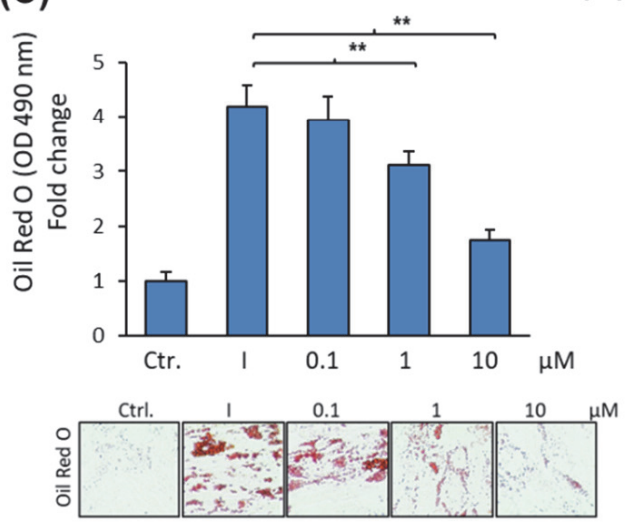

(B)

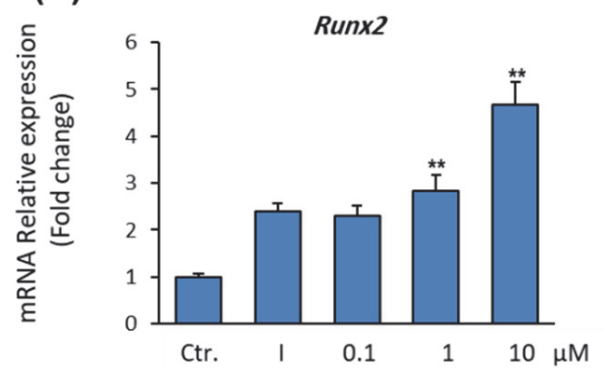

(E)

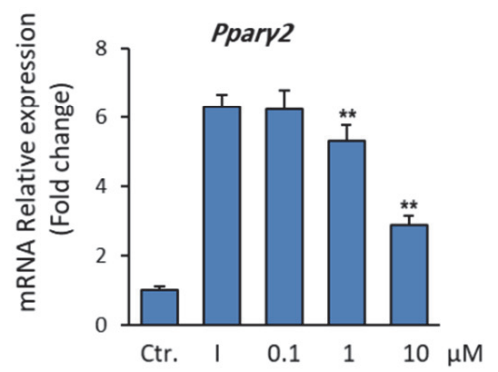

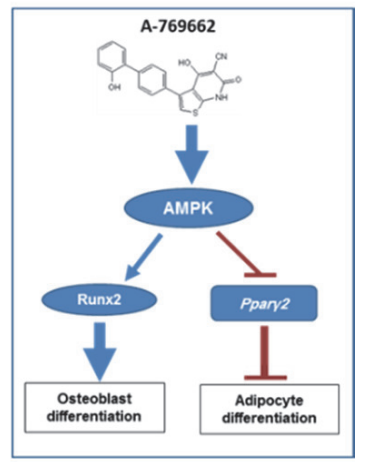

Fig. 6. A-769662 stimulates the differentiation of hBMSCs toward osteoblast versus adipocyte lineages. (A) A-769662 promotes hBMSCs differentiation into osteoblast in dose-dependent manner as measured by ALP activity quantification after 7 days of osteogenic induction. (B) qPCR analysis of Runx2 in mBMSCs treated with different concentrations of A-769662 after 7 days of osteogenic induction. (C) A-769662 suppresses adipogenesis of hBMSCs in dose-dependent manner as measured by reduced Oil red O quantification after 12 days of adipogenic induction. Staining images were presented. (D) qPCR analysis of Ppary2 gene expression in mBMSCs induced to adipocyte differentiation in the presence of different concentrations of A-769662. (E) Schematic diagram for the mode of action on A-769662 in promoting osteogenesis in BMSCs via activating AMPK signaling and targeting the upregulation of Runx2 and inhibition of Ppary2; ** $p<0.005$ compared to I+DMSO. Value of each experiment is mean \pm SD of independent triplicates.

rapeutic drug for promoting bone formation via acting directly on stimulating the BMSCs differentiation into osteoblast. Further pre-clinical studies are required to provide in vivo date on the potential therapeutic effect of A-769662 on bone in a bone loss murine model.

\section{Conclusions}

In this report, we have demonstrated the effect of the AMPK activator A-769662 in promoting the differentiation fate of murine and human BMSCs into osteoblastic versus adipocytic cell lineage. The regulatory effect of A-769662 on BMSCs differentiation is mediated via an AMPK-dependent mechanism and by targeting the upregulation of Runx2 and downregulation of Ppary2 gene expression (Fig. 6E). Thus, our data identified A-769662 as a possible osteo-anabolic drug that can enhance bone formation in osteoporotic patients via directing the differentiation of BMSCs into osteoblasts versus adipocytes.

\section{Availability of data and materials}

All materials are available via the corresponding author.

\section{Conflict of interests}

The authors declare that they have no competing interests.

\section{Funding}

This work was funded by the Deanship of Scientific Research at King Faisal University, Saudi Arabia (Grant No. 180076).

\section{Authors' contributions}

BMA conceived and designed the project, and drafted the manuscript. BMA and AMZ performed experiments, data analysis, and revisions of the article, gave final approval of the version, and agreed to all aspects of the work.

\section{Acknowledgements}

The Authors acknowledge the Deanship of Scientific Research at King Faisal University for the financial support under the annual research project (Grant No. 180076). 


\section{References}

Abdallah BM, Kassem M (2008). Human mesenchymal stem cells: from basic biology to clinical applications. Gene Ther. 15(2): 109-116. DOI: 10.1038/sj.gt.3303067.

Abdallah BM, Alzahrani AM, Kassem M (2018). Secreted Clusterin protein inhibits osteoblast differentiation of bone marrow mesenchymal stem cells by suppressing ERK1/2 signaling pathway. Bone 110: 221-229. DOI: 10.1016/j.bone.2018.02.018.

Abdallah BM, Jafari A, Zaher W, Qiu W, Kassem M (2015). Skeletal (stromal) stem cells: an update on intracellular signaling pathways controlling osteoblast differentiation. Bone 70: 28-36. DOI: 10.1016/j.bone.2014.07.028.

Amantea CM, Kim WK, Meliton V, Tetradis S, Parhami F (2008). Oxysterol-induced osteogenic differentiation of marrow stromal cells is regulated by Dkk-1 inhibitable and PI3-kinase mediated signaling. J Cell Biochem 105(2): 424-436. DOI: 10.1002/ jcb. 21840

Babkov DA, Zhukowskaya ON, Borisov AV, Babkova VA, Sokolova EV, Brigadirova AA, et al. (2019). Towards multi-target antidiabetic agents: Discovery of biphenyl-benzimidazole conjugates as AMPK activators. Bioorg Med Chem Lett 29(17): 2443-2447. DOI: 10.1016/j.bmcl.2019.07.035.

Bennett CN, Longo KA, Wright WS, Suva LJ, Lane TF, Hankenson KD, MacDougald OA (2005). Regulation of osteoblastogenesis and bone mass by Wnt10b. Proc Natl Acad Sci U S A 102(9): 3324-3329. DOI: 10.1073/pnas.0408742102.

Bianco P, Robey PG (2015). Skeletal stem cells. Development 142(6): 1023-1027. DOI: 10.1242/dev.102210.

Chava S, Chennakesavulu S, Gayatri BM, Reddy ABM (2018). A novel phosphorylation by AMP-activated kinase regulates RUNX2 from ubiquitination in osteogenesis over adipogenesis. Cell Death Dis 9(7): 754. DOI: 10.1038/s41419-018-0791-7.

Chen G, Deng C, Li Y-P (2012). TGF-beta and BMP signaling in osteoblast differentiation and bone formation. Int J Biol Sci 8(2): 272-288. DOI: 10.7150/ijbs.2929.

Chen SC, Brooks R, Houskeeper J, Bremner SK, Dunlop J, Viollet B, et al. (2017). Metformin suppresses adipogenesis through both AMP-activated protein kinase (AMPK)-dependent and AMPKindependent mechanisms. Mol Cell Endocrinol 440: 57-68. DOI: 10.1016/j.mce.2016.11.011.

Cool B, Zinker B, Chiou W, Kifle L, Cao N, Perham M, et al. (2006). Identification and characterization of a small molecule AMPK activator that treats key components of type 2 diabetes and the metabolic syndrome. Cell Metab 3(6): 403-416. DOI: 10.1016/j. cmet.2006.05.005.

Corton JM, Gillespie JG, Hawley SA, Hardie DG (1995). 5-aminoimidazole-4-carboxamide ribonucleoside. A specific method for activating AMP-activated protein kinase in intact cells? Eur J Biochem 229(2): 558-565. DOI: 10.1111/j.14321033.1995.tb20498.x.

Day EA, Ford RJ, Steinberg GR (2017). AMPK as a Therapeutic Target for Treating Metabolic Diseases. Trends Endocrinol Metab 28(8): 545-560. DOI: 10.1016/j.tem.2017.05.004.

de Meester C, Timmermans AD, Balteau M, Ginion A, Roelants V, Noppe G, et al. (2014). Role of AMP-activated protein kinase in regulating hypoxic survival and proliferation of mesenchymal stem cells. Cardiovasc Res 101: 20-29. DOI: 10.1093/cvr/cvt227.

Ge C, Xiao G, Jiang D, Franceschi RT (2007). Critical role of the extracellular signal-regulated kinase-MAPK pathway in osteoblast differentiation and skeletal development. J Cell Biol 176(5): 709-718. DOI: 10.1083/jcb.200610046.

Gimble JM, Zvonic S, Floyd ZE, Kassem M, Nuttall ME (2006). Playing with bone and fat. J Cell Biochem 98(2): 251-266. DOI: $10.1002 / j c b .20777$.

Goransson O, McBride A, Hawley SA, Ross FA, Shpiro N, Foretz M, et al. (2007). Mechanism of action of A-769662, a valuable tool for activation of AMP-activated protein kinase. J Biol Chem 282(45): 32549-32560. DOI: 10.1074/jbc.M706536200.

Hardie DG (2007). AMP-activated protein kinase as a drug target. Annu Rev Pharmacol Toxicol 47: 185-210. DOI: 10.1146/annurev. pharmtox.47.120505.105304.
Hardie DG (2014). AMP-activated protein kinase: maintaining energy homeostasis at the cellular and whole-body levels. Annu Rev Nutr 34: 31-55. DOI: 10.1146/annurev-nutr-071812-161148.

Hardouin P, Rharass T, Lucas S (2016). Bone Marrow Adipose Tissue: To Be or Not To Be a Typical Adipose Tissue? Front Endocrinol (Lausanne) 7: 85. DOI: 10.3389/fendo.2016.00085.

Jafari A, Siersbaek MS, Chen L, Qanie D, Zaher W, Abdallah BM, Kassem M (2015). Pharmacological Inhibition of Protein Kinase G1 Enhances Bone Formation by Human Skeletal Stem Cells Through Activation of RhoA-Akt Signaling. Stem Cells 33(7): 2219-2231. DOI: 10.1002/stem.2013.

Kanazawa I, Yamaguchi T, Yano S, Yamauchi M, Sugimoto T (2009). Activation of AMP kinase and inhibition of Rho kinase induce the mineralization of osteoblastic MC3T3-E1 cells through endothelial NOS and BMP-2 expression. Am J Physiol Endocrinol Metab 296(1): E139-146. DOI: 10.1152/ajpendo.90677.2008.

Kanno T, Takahashi T, Tsujisawa T, Ariyoshi W, Nishihara T (2007). Mechanical stress-mediated Runx2 activation is dependent on Ras/ERK1/2 MAPK signaling in osteoblasts. J Cell Biochem 101(5): 1266-1277. DOI: 10.1002/jcb.21249.

Kim E-K, Lim S, Park J-M, Seo JK, Kim JH, Kim KT, et al. (2012). Human mesenchymal stem cell differentiation to the osteogenic or adipogenic lineage is regulated by AMP-activated protein kinase. J Cell Physiol 227(4): 1680-1687. DOI: 10.1002/jcp.22892.

Kim J, Yang G, Kim Y, Kim J, Ha J (2016). AMPK activators: mechanisms of action and physiological activities. Exp Mol Med 48: e224. DOI: 10.1038/emm.2016.16.

Li Y, Jin D, Xie W, Wen L, Chen W, Xu J, et al. (2018). PPAR-gamma and Wnt Regulate the Differentiation of MSCs into Adipocytes and Osteoblasts Respectively. Curr Stem Cell Res Ther 13(3): 185-192. DOI: 10.2174/1574888X12666171012141908.

Luby AO, Ranganathan K, Lynn JV, Nelson NS, Donneys A, Buchman SR (2019). Stem Cells for Bone Regeneration: Current State and Future Directions. J Craniofac Surg 30(3): 730-735. DOI: 10.1097/SCS.0000000000005250.

Mihaylova MM, Shaw RJ (2011). The AMPK signalling pathway coordinates cell growth, autophagy and metabolism. Nat Cell Biol 13(9): 1016-1023. DOI: $10.1038 /$ ncb2329.

Molinuevo MS, Schurman L, McCarthy AD, Cortizo AM, Tolosa MJ, Gangoiti MV, et al. (2010). Effect of metformin on bone marrow progenitor cell differentiation: in vivo and in vitro studies. $\mathrm{J}$ Bone Miner Res 25(2): 211-221. DOI: 10.1359/jbmr.090732.

Rharass T, Lucas S (2018). MECHANISMS IN ENDOCRINOLOGY: Bone marrow adiposity and bone, a bad romance? Eur J Endocrinol 179(4): R165-R182. DOI: 10.1530/EJE-18-0182.

Sanders MJ, Ali ZS, Hegarty BD, Heath R, Snowden MA, Carling D (2007). Defining the mechanism of activation of AMP-activated protein kinase by the small molecule A-769662, a member of the thienopyridone family. J Biol Chem 282(45): 32539-32548. DOI: $10.1074 /$ jbc.M706543200.

Scott JW, Ling N, Issa SM, Dite TA, O’Brien MT, Chen ZP, et al. (2014). Small molecule drug A-769662 and AMP synergistically activate naive AMPK independent of upstream kinase signaling. Chem Biol 21(5): 619-627. DOI: 10.1016/j.chembiol.2014.03.006.

Sedlinsky C, Molinuevo MS, Cortizo AM, Tolosa MJ, Felice JI, Sbaraglini ML, et al. (2011). Metformin prevents anti-osteogenic in vivo and ex vivo effects of rosiglitazone in rats. Eur J Pharmacol 668(3): 477-485. DOI: 10.1016/j.ejphar.2011.07.033.

Shah M, Kola B, Bataveljic A, Arnett TR, Viollet B, Saxon L, et al. (2010). AMP-activated protein kinase (AMPK) activation regulates in vitro bone formation and bone mass. Bone 47(2): 309-319. DOI: 10.1016/j.bone.2010.04.596.

Shen W, Chen J, Gantz M, Punyanitya M, Heymsfield SB, Gallagher D, et al. (2012). Ethnic and sex differences in bone marrow adipose tissue and bone mineral density relationship. Osteoporos Int 23(9): 2293-2301. DOI: 10.1007/s00198-0111873-x.

Suh JM, Gao X, McKay J, McKay R, Salo Z, Graff JM (2006). Hedgehog signaling plays a conserved role in inhibiting fat formation. Cell Metab 3(1): 25-34. DOI: 10.1016/j. cmet.2005.11.012.

Taipaleenmaki H, Abdallah BM, Aldahmash A, Saamanen AM, Kassem M (2011). Wnt signalling mediates the cross-talk between 
bone marrow derived pre-adipocytic and pre-osteoblastic cell populations. Exp Cell Res 317(6): 745-756. DOI: 10.1016/j. yexcr.2010.12.015

Tamargo-Gomez I, Marino G (2018). AMPK: Regulation of Metabolic Dynamics in the Context of Autophagy. Int J Mol Sci 19(12): 3812. DOI: $10.3390 /$ ijms19123812.

Thirupathi A, Chang Y-Z (2019). Role of AMPK and its molecular intermediates in subjugating cancer survival mechanism. Life Sci 227: 30-38. DOI: 10.1016/j.lfs.2019.04.039.

Towler MC, Hardie DG (2007). AMP-activated protein kinase in metabolic control and insulin signaling. Circ Res 100(3): 328-341. DOI: 10.1161/01.RES.0000256090.42690.05.

Wang S, Song P, Zou M-H (2012). AMP-activated protein kinase, stress responses and cardiovascular diseases. Clin Sci (Lond) 122(12): 555-573. DOI: 10.1042/CS20110625.

Wu L, Zhang L, Li B, Jiang H, Duan Y, Xie Z, et al. (2018). AMPActivated Protein Kinase (AMPK) Regulates Energy Metabolism through Modulating Thermogenesis in Adipose Tissue. Front Physiol 9: 122. DOI: 10.3389/fphys.2018.00122.

Zhao L, Hantash BM (2011). TGF-beta1 regulates differentiation of bone marrow mesenchymal stem cells. Vitam Horm 87: 127-141. DOI: 10.1016/B978-0-12-386015-6.00042-1.

Zheng C, Chen J, Liu S, Jin Y (2019). Stem cell-based bone and dental regeneration: a view of microenvironmental modulation. Int J Oral Sci 11(3): 23. DOI: 10.1038/s41368-019-0060-3.

Zhou Y, Wang D, Zhu Q, Gao X, Yang S, Xu A, Wu D (2009). Inhibitory effects of A-769662, a novel activator of AMP-activated protein kinase, on 3T3-L1 adipogenesis. Biol Pharm Bull 32(6): 993-998. DOI: 10.1248/bpb.32.993.

Zhu Y, Zhou J, Ao R, Yu B (2014). A-769662 protects osteoblasts from hydrogen dioxide-induced apoptosis through activating of AMP-activated protein kinase (AMPK). Int J Mol Sci 15(6): 11190-11203. DOI: 10.3390/ijms150611190. 


\section{Supplementary materials}

Suppl. Table 1. List of primers used for qPCR

\begin{tabular}{|c|c|c|}
\hline Gene name & Forward primer 5'-3' & Reverse primer 5'-3' \\
\hline$\beta$-Actin & GAT ATC GCT GCG CTG GTC GTC & ACG CAG CTC ATT GTA GAA GGT GTG G \\
\hline Runx2 & AGC AAC AGC AAC AAC AGC AG & GTA ATC TGA CTC TGT CCT TG \\
\hline Ocn & CAG ACA AGT CCC ACA CAG CA & CTT TAT TTT GGA GCT GCT GT \\
\hline Alp & GCC CTC TCC AAG ACA TAT A & CCA TGA TCA CGT CGA TAT CC \\
\hline Osx & TATGCTCCGACCTCCTCAAC & AATAGGATTGGGCAGAAAG \\
\hline Col1a1 & GGT GAA CAG GGT GTT CCT GG & TTC GCA CCA GGT TGG CCA TC \\
\hline Opn & GAA ACT CTT CCA AGC AAT TC & GGA CTA GCT TGT CCT TGT GG \\
\hline Hprt & TCAGTCAACGGGGGACATAAA & GGGGCTGTACTGCTTAACCAG \\
\hline Ppary2 & GGG TCA GCT CTT GTG AAT GG & CTG ATG CAC TGC CTA TGA GC \\
\hline C/ebpa & AAG CCA AGA AGT CGG TGG A & CAG TCC ACG GCT CAG CTG TTC \\
\hline$a P 2$ & CAA AAT GTG TGA TGC CTT TGT G & CTC TTC CTT TGG CTC ATG CC \\
\hline Lpl & CTGCTGGCGTAGCAGGAAGT & GCTGGAAAGTGCCTCCATTG \\
\hline
\end{tabular}

(A)

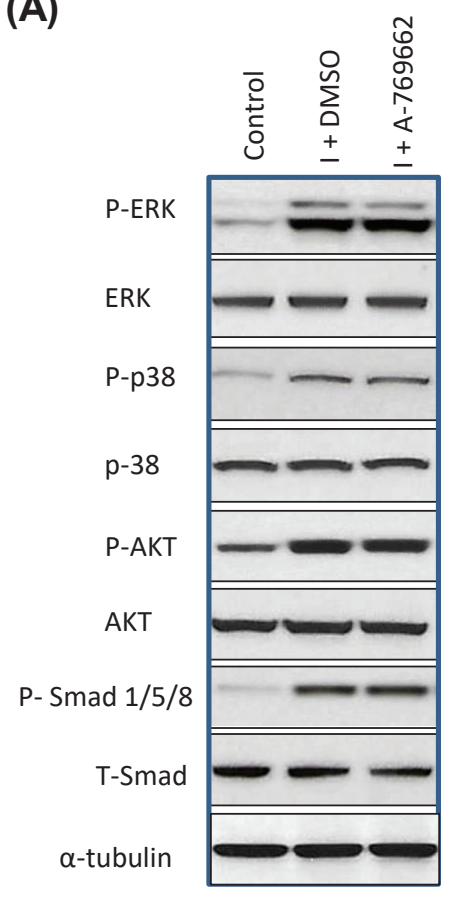

(B)

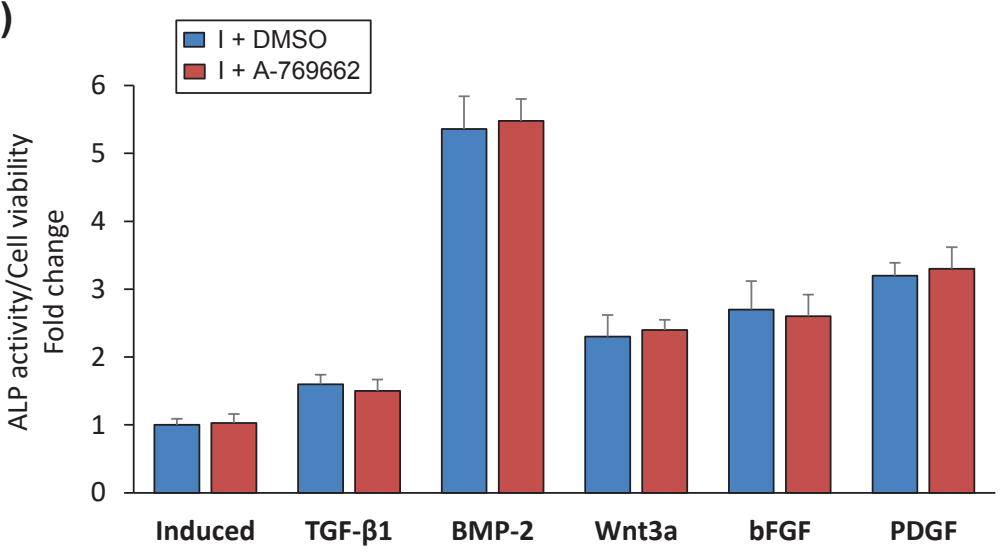

(A) Western blot analysis of the effect of A-769662 on the activation of several osteogenic signaling molecules including P-ERK, P-p38, P-AKT, and P-Smad 1/5/8 during osteoblast differentiation of mBMSCs. Cells were induced to differentiate into osteoblast as described in M\&M in the absence (I+DMSO) or the presence of A-769662 $(10 \mu \mathrm{M})$ for 3 days. For Smad phosphorylation, cells were induced with BMP2 (110 ng/ml). (B) Effect of A-769662 on the stimulatory effect of some osteogenic signaling molecules on the osteoblast differentiation of mBMSCs. Cells were induced to osteoblast differentiation without or with A-769662 $(10 \mu \mathrm{M})$ in the presence of TGFß1 $(10 \mathrm{ng} / \mathrm{ml})$, BMP2 $(100 \mathrm{ng} / \mathrm{ml})$, Wnt3a $(10$ $\mathrm{ng} / \mathrm{ml})$, PDGF $(100 \mathrm{ng} / \mathrm{ml}) \mathrm{bFGF}(100 \mathrm{ng} / \mathrm{ml})$ for 7 days. Values are mean $\pm \mathrm{SD}$ of three independent experiments.

Suppl. Fig. 1. Effect of A-769662 on osteogenic signaling pathways 